\title{
Retail Chicken Carcasses as a Reservoir of Antimicrobial- Resistant Escherichia coli
}

\author{
Nahla O. Eltai ${ }^{1}$, Hadi M. Yassine ${ }^{1}$, Sara H. Al-Hadidi1, Tahra El-Obeid ${ }^{2}$, Asmaa A. Al Thania ${ }^{1}$, Walid Q. Alali
}

${ }^{1}$ Biomedical Research Center, Qatar University, ${ }^{2}$ Department of Health Nutrition, College of Health Sciences, Qatar University, ${ }^{3}$ Department of Epidemiology and Biostatistics, Faculty of Public Health, Kuwait University, Safat, Kuwait.

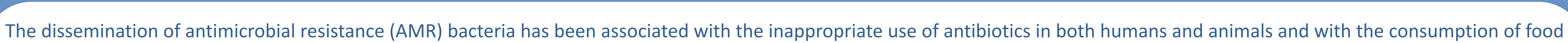

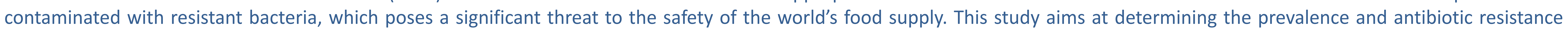

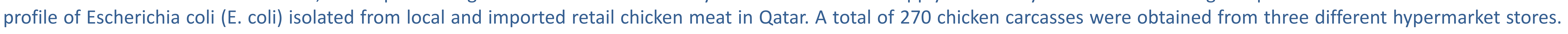

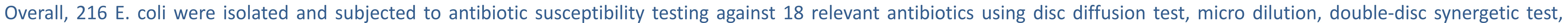

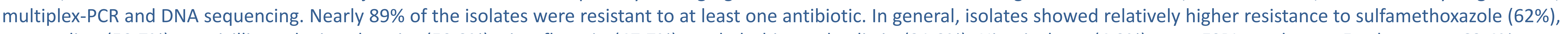

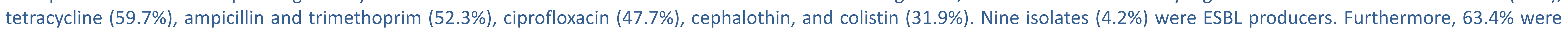

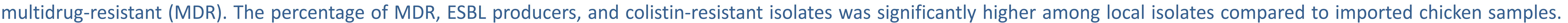

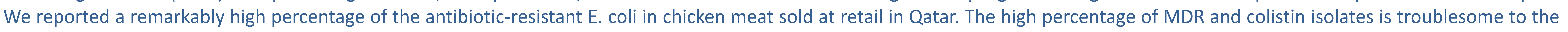

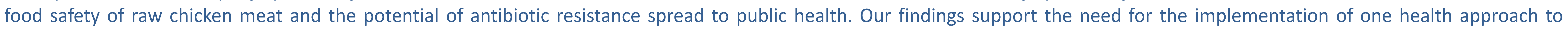
address the spread of antimicrobial resistance and the need for a collaborative solution.
\end{abstract}

\section{BACKGROUND}

The dissemination of antimicrobial resistance (AMR) bacteria has been associated with the inappropriate use of antibiotics in both humans and animals and with the consumption of food contaminated with resistant bacteria. In particular, the use of antibiotics as prophylactic and growth promotion purposes in food-producing animals has rendered many of the antibiotics ineffective. The increased global prevalence of AMR poses a significant threat to the safety world's food supply. Furthermore, studies have revealed that antibiotic resistant Escherichia coli (E. coli) from food sources, such as poultry and poultry products, can spread to humans and potentially colonize human gut. Moreover, E. coli can transfer their resistant genes to pathogenic bacteria such as Salmonella in different environment. Additionally, resistant $E$. coli can cause urinary tract infection, meningitis, peritonitis, and septicemia. Escherichia coli is generally used as a sentinel for monitoring antibiotic resistance.

In Qatar, multi-drug resistant (MDR) particularly in gram-negative strains including $E$. coli and Klebsiella as well as colistin resistant bacteria has been reported in both humans and animals (25-27). However, there is no information available on antibiotic resistant $E$. coli on raw chicken meat at the retail level in Qatar. Here we report on the prevalence of antibiotic resistant $E$. coli isolates found on chicken carcasses sold at retaillevel in Qatar.

\section{OBJECTIVES}

This study aims at determining the prevalence of antibiotic-resistant $E$. coli isolated from local and imported retail chicken meat in Qatar.

Characterizing antibiotic resistance at phenotypic and genotypic levels.

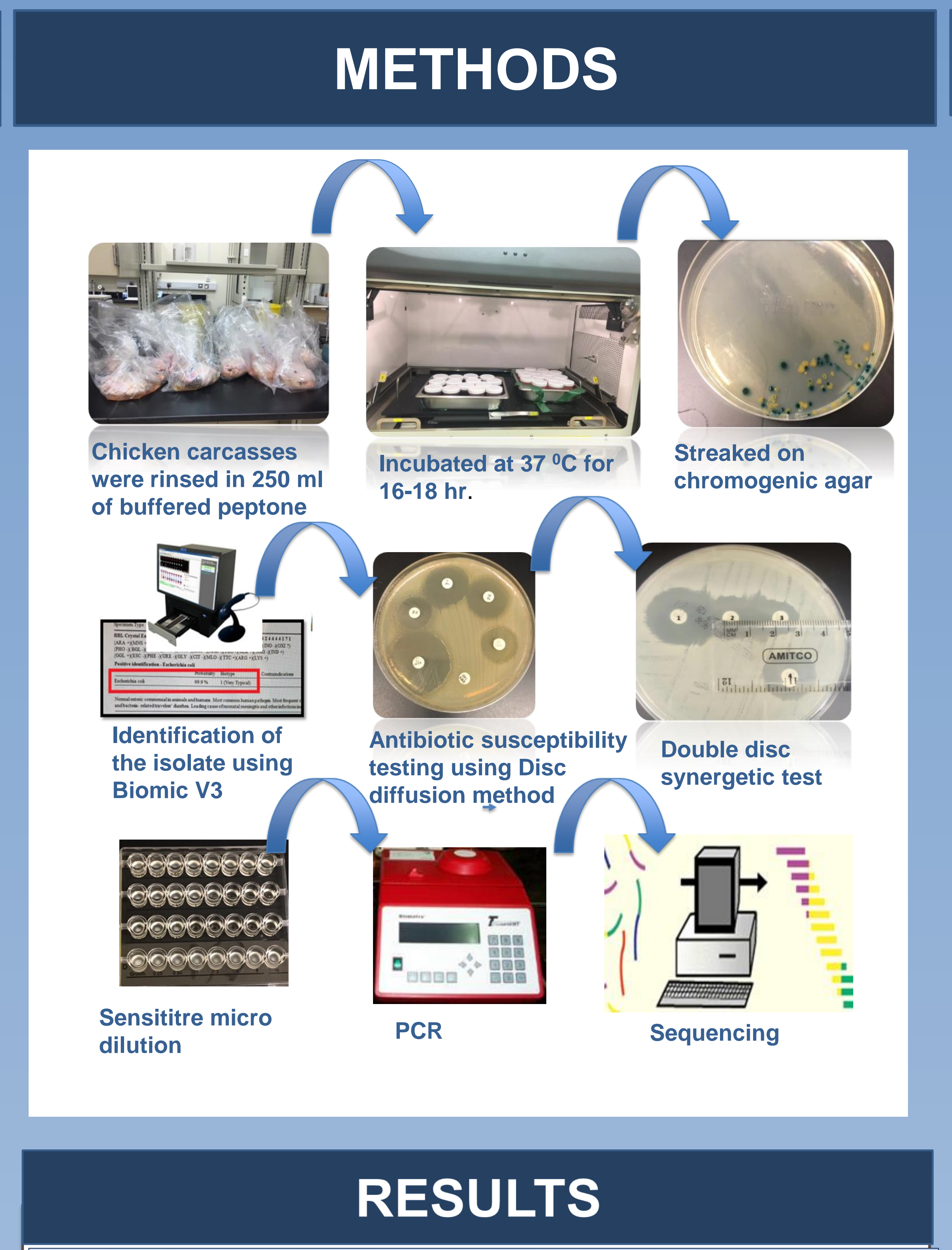

Fig. 1. Prevalence of antibiotic resistant $E$. coli isolates $(n=216)$ by storage temperature and source in Qatar*

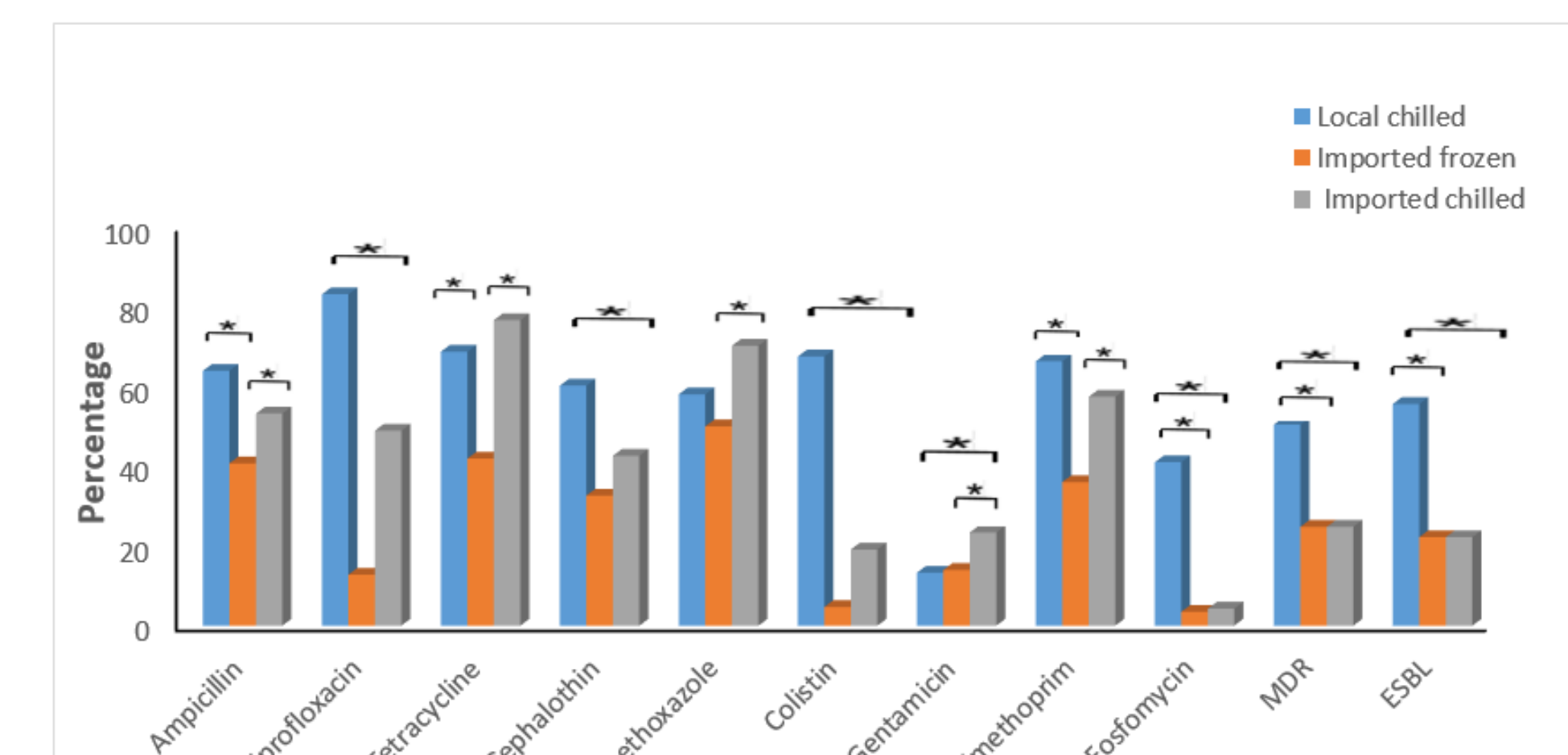

* percentages are shown only for resistance with significant differences by storage temperature and source

Significant difference $P<0.05$ (Person Chi-square test); ESBL: Extended spectrum beta lactamase producer; MDR: Multi Drug Resistant

Fig.2: Detection of bla $a_{\mathrm{SHV}}, b_{\mathrm{TEM}}$ and bla $a_{\mathrm{CTX}-\mathrm{M}-\mathrm{G}}$ $(1,2)$ antibiotic resistance genes in $E$. coli isolated from retail chicken carcasses*

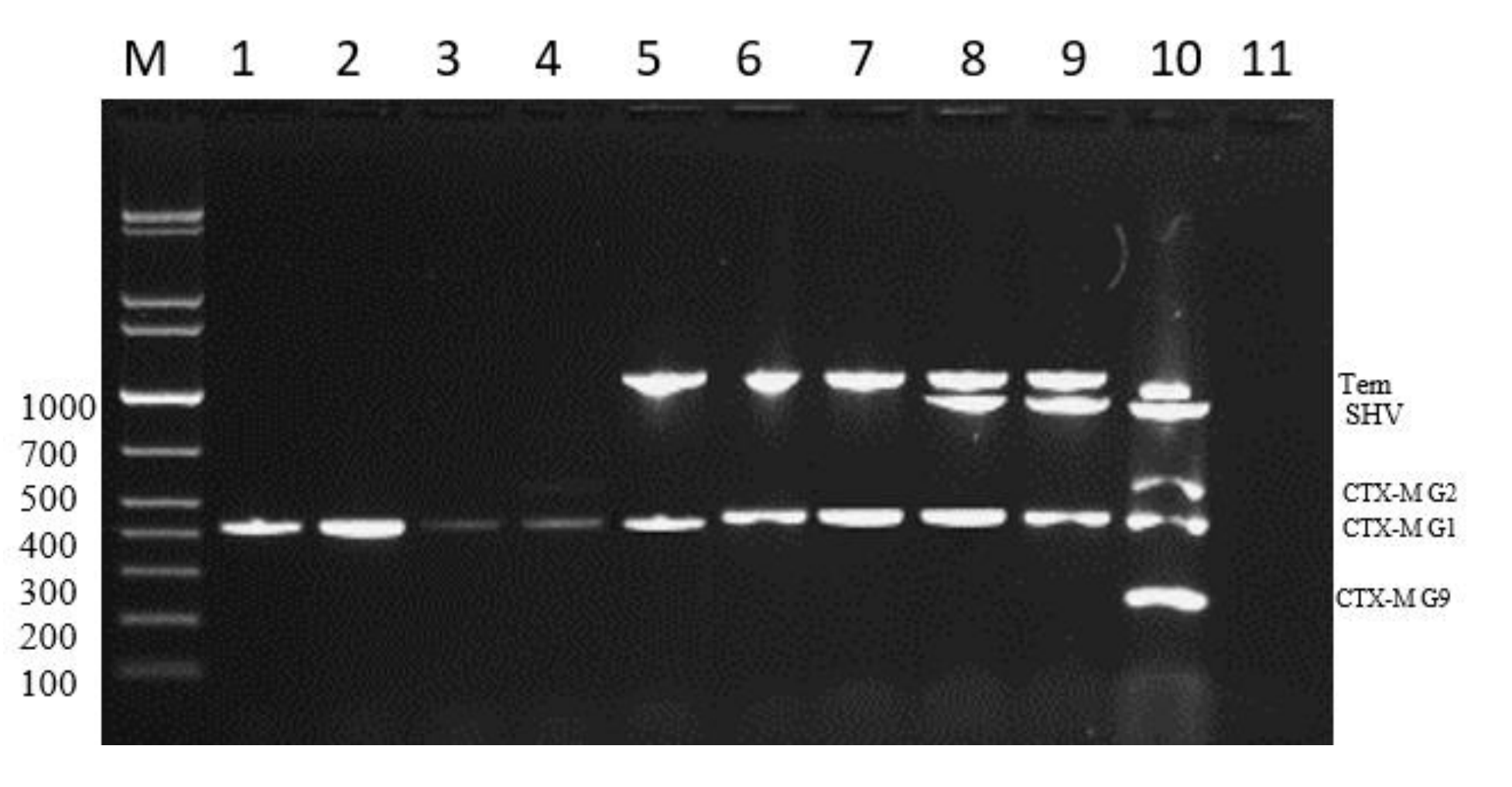

This figure displays different isolates harboring different bla genes. Multiplex PCR was performed for detection of CTX-M groups and PCR for detection of TEM and SHV. The amplification products of each isolate were run on the same lane for detection of bla genes. Lanes 1 to $3: b / a_{\text {CTX-M }}$
Lane 4: bla $a_{\text {CTX }}$

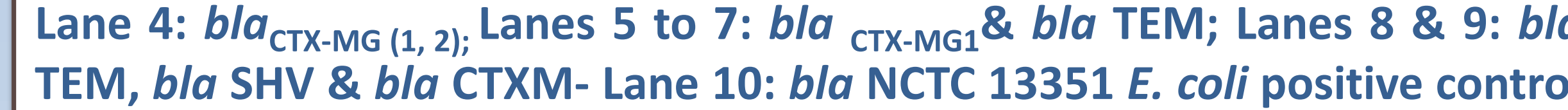
for blaTEM, NCTC $13368 \mathrm{~K}$. pneumonia positive control for blaSHV, NCTC for blaTEM, NCTC $13368 \mathrm{~K}$. pneumonia positive control for blaSHV, NCTC
$13461 \mathrm{E}$. coli positive control for bla $a_{\text {cTX-MGi; }}$ NCTC $13462 \mathrm{E}$. coli positive Lane 11: ATCC 25922 E. coli negative E.

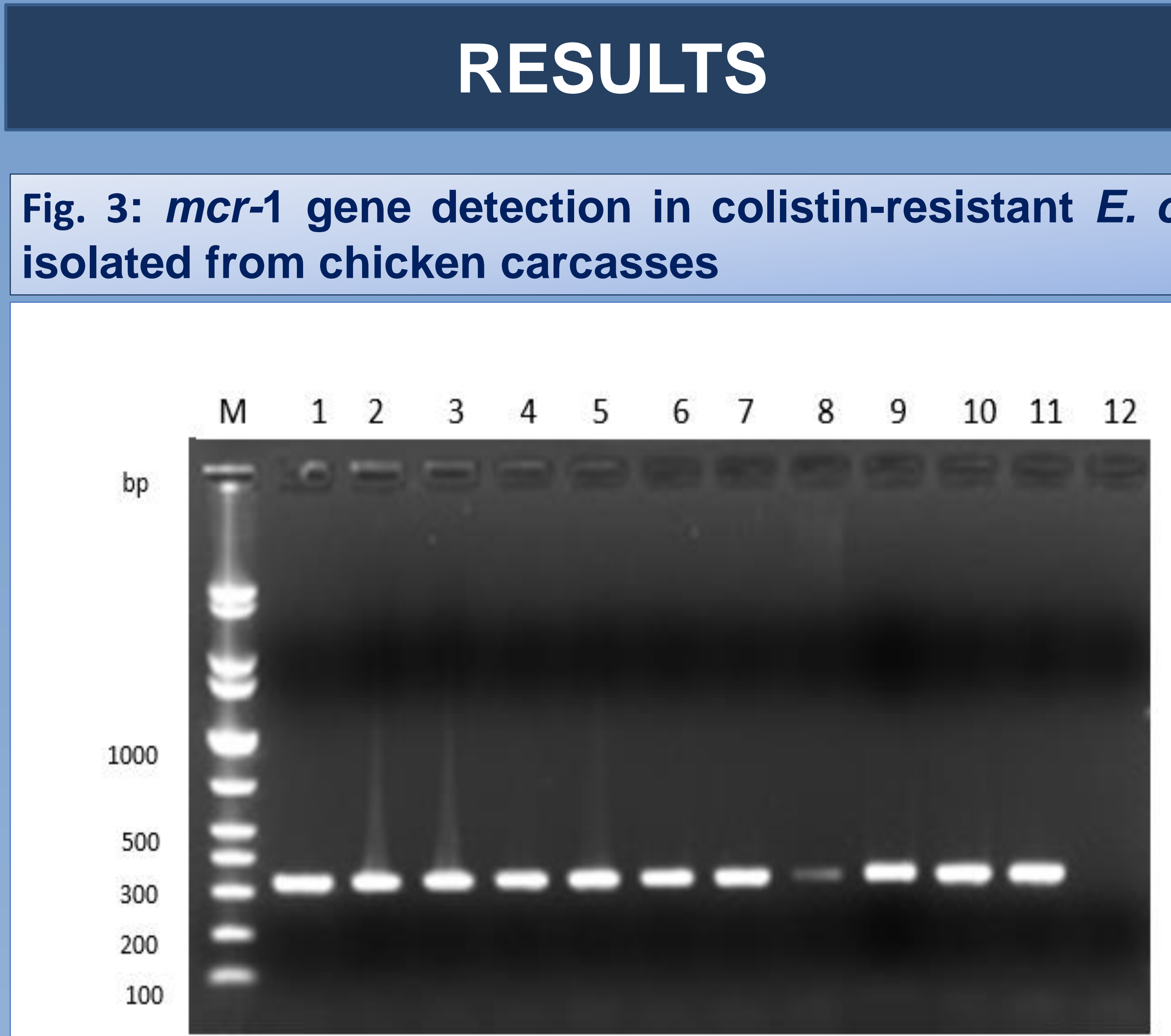

Representative samples are shown. Multiplex PCR was performed for detection of $m c r-1,2,3,4,5$ genes. Resistance to colistin in 69 isolates was confirmed by PCR amplification of 320 bp fragment of mcr-1 gene. Lanes 1-11: PCR products of part of mcr-1 gene (320pb); Lane 12: ATCC 25922 E. coli (sensitive strain. M: molecular size (weight) standard marker; bp: base-pairs. The following illustrates the sequence of $m c r-1$ PCR product:

(TATAAAAACGTTCTGACGCGACCGCCATCTTACCTTTTTTGATAAAAT CAGCCAAACCTATCCCATCGCGGACAATCTCGGCTTTGTGCTGACGA TCGCTGTCGTGCTCTTTGGCGCGATGCTACTGATCACCACGCTGTTA TCATCGTATCGCTATGTGCTAAAGCCTGTGTTGATTTTGCTATTAATCA TGGGCGCGGTGACCAGTTATTTTACTGACACTTATGGCACGGTCTAT GATACGACCATGCTCCAAAATGCCCTACAGACCGACCAAGCCGAGA CCAAGGATCTGA).

\section{CONCLUSIONS}

We reported a remarkably high percentage of the antibiotic-resistant E. coli in chicken meat sold at retail in Qatar. The high percentage of MDR and colistin isolates is troublesome to the food safety of raw chicken meat and the potential of antibiotic resistance spread to public health. Our findings support the need for the implementation of one health approach to address the spread of antimicrobial resistance and the need for a collaborative solution.

\section{REFERENCES}

Eltai NO, Abdfarag EA, Al-Romaihi H, Wehedy E, Mahmoud MH, Alawad OK, A mmensal Escherichia coli isolated from broiler chickens in Qatar. Journal of food protection 81:302-307.
Eltai NO, Al Thani AA, Al-Ansari K, Deshmukh AS, Wehedy E, Al-Hadidi SH Eltai NO, AI Thani AA, Al-Ansari K, Deshmukh AS, Wehedy E, Al-Hadidi SH,
Yassine HM. 2018. Molecular characterization of extended spectrum $\beta$ Yassine HM. 2018. Molecular characterization of extended spectrum $\beta$ -
lactamases enterobacteriaceae causing lower urinary tract infection among lactamases enterobacteriaceae causing lower urinary tract infection among
pediatric population. Antimicrobial Resistance \& Infection Control 7:90. Ahmed MAS, Bansal D, Acharya A, Elmi AA, Hamid JM, Ahmed AMS, Chandra molecular epidemiology of extended-spectrum beta-lactamase-producing Enterobacteriaceae from intensive care units at Hamad Medical Corporation, Qatar. Antimicrobial resistance and infection control 5

\section{Acknowledgements}

This work was funded by BCR grant 